\title{
Characterization of structural change in rail surface using advanced automatic crystallographic orientation microscopy
}

\author{
J. Wu ${ }^{1}$, R. H. Petrov ${ }^{1,2}, \mathrm{~S} . \mathrm{Li}^{3}$, Z. $\mathrm{Li}^{3}, \mathrm{~S} . \mathrm{Godet}^{4}$, \\ L. Malet ${ }^{4} \&$ J. Sietsma ${ }^{1,2}$ \\ ${ }^{1}$ Department of Materials Science and Engineering, \\ Delft University of Technology, The Netherlands \\ ${ }^{2}$ Department of Materials Science and Engineering, \\ Ghent University, Belgium \\ ${ }^{3}$ Section of Railway Engineering, Faculty of Civil Engineering and \\ Geoscience, Delft University of Technology, The Netherlands \\ ${ }^{4}$ Matters and Materials Department, \\ Universite Libre de Bruxelles, Belgium
}

\begin{abstract}
Surface structural changes, formed during wheel/rail contact, in R260Mn grade rails were investigated by electron backscatter diffraction (EBSD), transmission Kikuchi diffraction (TKD) in scanning electron microscope (SEM) and automatic crystal orientation mapping in transmission electron microscope (ACOM-TEM). Grain fragmentation and refinement of ferrite are characterized by all applied methods as well as grain alignment towards traffic direction. Substructures, having misorientation lower than $5^{\circ}$, are identified by kernel average misorientation (KAM). Detection of retained austenite indicates martensitic nature of the structural change observed in the heavily deformed surface layer.

Keywords: surface structural change, brown etching layer (BEL), pearlitic rails, electron backscattered diffraction (EBSD), transmission Kikuchi diffraction (TKD), automatic crystal orientation mapping in transmission electron microscope (ACOM-TEM), martensite, retained austenite, Kurdjumov-Sachs $(K-S)$ orientation relationship.
\end{abstract}




\section{Introduction}

Due to wheel/rail contact, a newly formed layer is commonly observed on the surface of various pearlitic grade rail steels [1-7]. This layer etches very hard and usually appears in white after etching by $2 \%$ Nital $\left(2\right.$ vol. $\% \mathrm{HNO}_{3}$ in $\left.\mathrm{C}_{2} \mathrm{H}_{5} \mathrm{OH}\right)$. Due to this etching response it is known as white etching layer (WEL). Until now, the formation mechanism for the WEL remains unclear and there are two hypotheses that are widely accepted. The first one proposes that the formation of the WEL is associated with martensitic transformation. Supporting points for this hypothesis are that: (i) tetragonality in iron ferrite is identified by peak splitting at peaks for $\{200\}_{\text {ferrite, }}$ using X-ray diffraction (XRD) $[3,5]$; (ii) the history of significant temperature rise, over $800^{\circ} \mathrm{C}$, during wheel/rail contact is supported by the detection of manganese diffusion using atom probe [6]; and (iii) the direct evidence of phase changes in the WEL is the detection of retained austenite, $c f$. by XRD by Wang et al. [5]. This evidence indicates martensitic WEL formed by phase transformation during frictional heating during the passing of trains and subsequent fast cooling. On the other hand, carbon supersaturated ferrite (martensite) fails to be detected by XRD in WEL in [7]. In addition, the high temperature necessary for pearlite to austenite transformation cannot always be supported. As an alternative, a hypothesis for cementite dissolution and fragmentation of ferritic grains into nano-size, induced by severe plastic deformation, is proposed to interpret the WEL phenomenon.

The above two hypotheses indicate different crystallographic orientations for the corresponding types of WEL. For the martensitic phase transformation, specific orientation relationship between martensite and retained austenite is expected. For example, Kurdjumov-Sachs $(\mathrm{K}-\mathrm{S})$ orientation relationship between martensite and retained austenite, $\{110\}_{\alpha}, / /\{111\}_{\gamma},<111>_{\alpha}, / /<101>_{\gamma}$, is widely identified in the lath martensite $[8,9]$. However, crystallographic orientation evolution in pearlitic ferrite after severe plastic deformation is different from that after martensitic phase transformation. For example, a $<110>$ type fibre textures is usually expected in pearlite after wire drawing [10].

Automatic orientation microscopy is suitable for differentiating the above mentioned hypotheses. The most widely used orientation based technique is the electron backscatter diffraction (EBSD) and it has been used for characterization of steels after plastic deformation and martensitic structures, $c f$. [9, 11]. EBSD is very sensitive to crystal orientation changes and the angular resolution can be down to $\sim 0.7^{\circ}$. Recently, automatic orientation microscopes with improved spatial resolution have been developed, such as transmission Kikuchi diffraction (TKD) in SEM [12], or automatic crystal orientation mapping in transmission electron microscope (ACOM-TEM) [13]. Due to the significantly reduced interacting volume of the sample with the electron beam, the spatial resolution is improved to down to several nanometres for TKD and ACOM-TEM. In this study, EBSD, TKD and ACOM-TEM are applied to characterize the observed surface structural change in severely deformed surface layer in rails. 


\section{Materials and methods}

A possible new type of WEL, which is etched brown colour [14], was investigated in this study, see Figure 1(a). It is named as brown etching layer (BEL), in order to be differentiated from the widely reported WEL. The notations of RD, ND and TD refer to the traffic direction (RD), rail normal direction (ND) and the transverse direction respectively (TD).

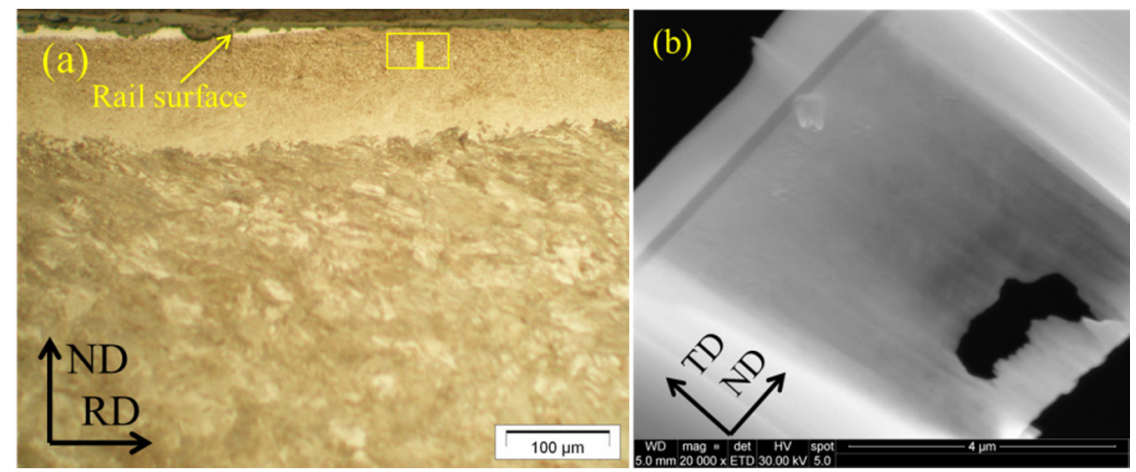

Figure 1: (a) Optical image of brown etching layer (BEL), which was studied in this work. The same BEL can also be found in [14]. The open square indicates the area of the EBSD scan; (b) A thin lamella extracted from the area indicated by the filled square in (a) by lift out method for TEM sample preparation with focuses ion beam (FIB) milling. The black zone in (b) is a hole which was made at the bottom of the lamella due to too long milling and the top layer is the deposited protective Pt layer.

EBSD characterization was done on the same plane as in Figure 1(a), namely $\mathrm{ND} \times \mathrm{RD}$ plane. The open square schematically indicates the position for EBSD scan. The specimen was prepared following the conventional routes for metallography characterization preparation till $1 \mu \mathrm{m}$ diamond paste. A further 40 minutes polishing using oxide polishing suspension (OPS) was applied to remove the deformation layer during sample preparation. The EBSD system attached to Quanta 450 scanning electron microscope (SEM) with field emission gun (FEG) was used to collect the data, under hexagonal scan mode with TSL-OIM ${ }^{\circledR}$ Data collection software. Accelerating voltage of $20 \mathrm{kV}$, working distance of $16 \mathrm{~mm}$ and step size of $50 \mathrm{~nm}$ were used for the data collection.

A thin lamella for TKD and ACOM-TEM characterizations (Figure 1(b)) was prepared by focus ion beam (FIB) milling in a FEI Nova 600 Nanolab Dual-Beam FIB system. The lamella was taken at $\sim 8 \mu \mathrm{m}$ away from the top surface, which is within the depth region for EBSD scan, (see filled and hollow squares in Figure 1(a)). The similar configuration settings for EBSD were used for the TKD measurements, except the sample holder. The specific TKD sample holder was pre-tilted minus $30^{\circ}$ and the stage was $20^{\circ}$ tilted away from EBSD CCD camera. 
Thus a total tilt of minus $10^{\circ}$ was used. Accelerating voltage of $30 \mathrm{kV}$, working distance of $5 \mathrm{~mm}$ and step size of $15 \mathrm{~nm}$ were used for the data collection.

ACOM-TEM was performed in a Philips CM20 TEM equipped with thermionic LaB6 electron gun. A camera length of $120 \mathrm{~mm}$ was used for the characterization. Accelerating voltage of $200 \mathrm{kV}$ and step size of $12 \mathrm{~nm}$ were used for data collection.

Crystallographic information was obtained by automatic indexing of diffraction patterns. For all measurements, BCC phase (ferrite) was indexed and FCC phase (austenite) was only indexed in ACOM-TEM measurements. All data were post processed with TSL-OIM ${ }^{\circledR}$ Data post-processing software (version 6.2).

\section{Results}

The studied brown etching layer (BEL) is a new type white etching layer (WEL) [14]. Unlike white colour of WEL, BEL etches white and brown under unpolarised light reflection, after etching with $2 \%$ Nital. Micro-hardness measurements show that the BEL is significantly softer than the reported WEL. In this study, BEL, observed in rail surface, was investigated.

As several parameters implemented in the TSL software were used for qualitative and quantitative characterization, a brief introduction to the meaning of these parameters should be given first: (i) Image quality (IQ). This parameter is used for qualitative description of strains in crystals, as it is affected by both crystal distortion and other factors, such as sample conditions. In general, higher IQ indicates lattice distortion which might be interpreted as less strain. (ii) Inverse pole figure (IPF). The parameter describes position of sample direction(s) relative to the crystal reference frame. (iii) Kernel average misorientation (KAM). This parameter characterizes the local misorientation. Within one grain, misorientation between kernel centre and the perimeter pixels are estimated and the average misorientation is assigned to the kernel centre pixel, provided a specific restricting conditions: a maximum angle, and the neighbouring pixels of the kernel (first, second, etc.). In this work the KAM was calculated using a max angle of $5^{\circ}$ and the first nearest neighbouring pixels for EBSD, $5^{\circ}$ and $3 \mathrm{rd}$ neighbour for TKD and the $5^{\circ}$ and 4 th neighbour for the ACOM-TEM. All pixels with misorientation larger than $5^{\circ}$ were excluded of the calculations.

Figure 2(a)-(c) shows the IQ map, the IPF parallel with RD direction and KAM map of EBSD scan on the selected area in Figure 1(a). In the IQ map (Figure 2(a)) fragmented grains can be observed and variations in the grey levels can be seen. The fragmented grains are also recognized in the IPF in Figure 2(b), as grains with different orientations are decorated by different colours. The grains closer to the rail surface are observably finer than those farther away from the rail surface. It was also observed that the grains are flattened and aligned towards the traffic direction, namely the RD in Figure 2. The KAM map, shown in Figure 2(c) displays the substructure within the grains indicated by the green lines. The high KAM pixels, with misorientation of $5^{\circ}$ appearing in red in Figure 2(c) correspond to the poor-indexed pixels at grain boundaries in Figure 2(b). 


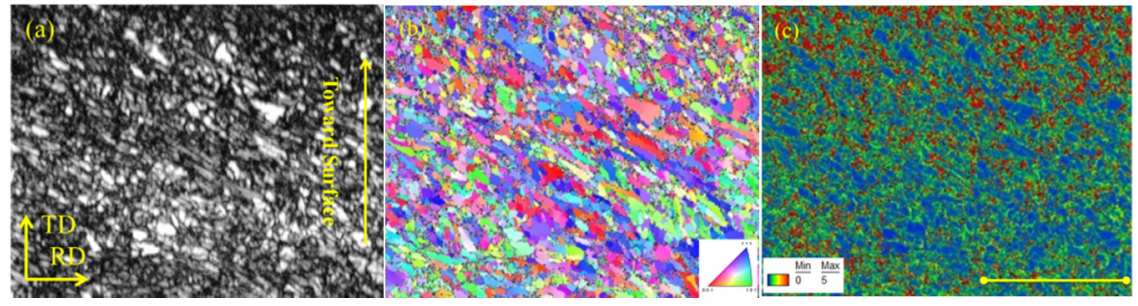

Figure 2: (a) IQ map. (b) IPF // RD direction and (c) KAM using $5^{\circ}$ and 1 st neighbour of EBSD scan on the selected area in Figure 1(a). The scale bar for all maps, shown in (c), is $10 \mu \mathrm{m}$.

Figure 3(a)-(c) shows the IQ map, IPF and KAM of a TKD scan on the thin FIB lamella. The IQ map in Figure 3(a) and IPF in Figure 3(b) display elongated and flattened grains. Combing grain morphology in Figure 3(a) and grains from EBSD scan in Figure 2(a) or Figure 2(b), it can be seen that the grains appear to have plate-like morphology from a three dimensional view. The darker areas close to the hole at the image bottom (Figure 3(a)) are due to fact that these areas are too thin to display Kikuchi diffraction patterns. KAM map in Figure 3(c) which is using the 3rd neighbour shows internal structure of the grains. The pixels with KAM misorientation of $5^{\circ}$, are the poor-indexed pixels in Figure 3(b). The misorientation profile along the dashed-line in Figure 3(a) will be shown and discussed in the subsequent section.
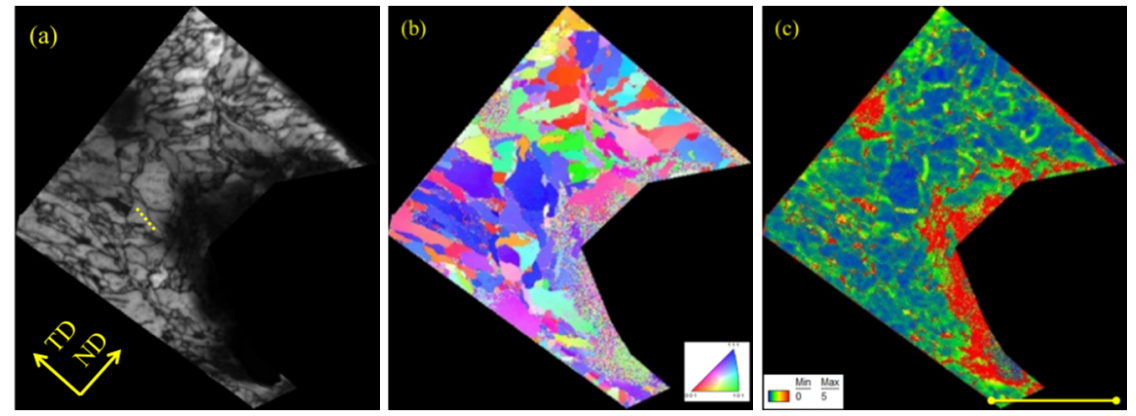

Figure 3: (a) IQ map; (b) IPF // RD direction; and (c) KAM using $5^{\circ}$ and 3 rd neighbour of TKD scan on FIB lamella. The scale bar in (c) is $2 \mu \mathrm{m}$.

Figure 4(a)-(c) shows the IQ map, IPF and KAM of BCC phase from ACOMTEM scan, on the same lamella that was used for TKD scan. The black zones within the specimen, Figure 4(a) and Figure 4(b) are the areas indexed as FCC phase. The area revealed by ACOM-TEM is noticeably larger than the one characterized by TKD. Similarly as for TKD in Figure 3(a)-(c), the IQ map in Figure 4(a) and IPF in Figure 4(b) show elongated and flattened grains. KAM map in Figure 4(c) was calculated using the 4th neighbour shows internal structure within grains. The dash-line shown in Figure 4(a) indicates the same grain in Figure 3(a). 

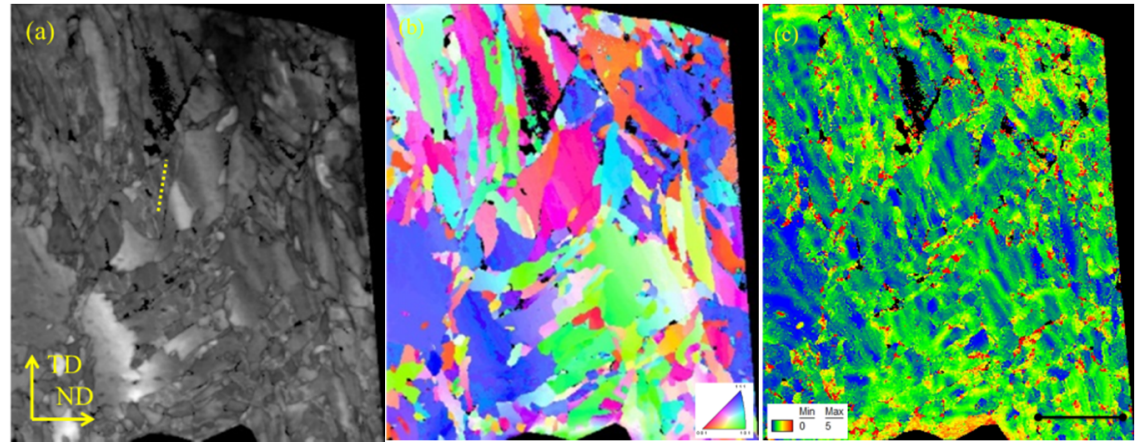

Figure 4: (a) IQ map; (b) IPF // RD direction; (c) KAM using 4th neighbour of ACOM-TEM scan on FIB lamella. The scale bar for all maps is shown in (c) and it is $1 \mu \mathrm{m}$.

The phase map of ferrite (BCC) and austenite (FCC) of the ACOM-TEM scan in Figure 4, is shown in Figure 5(a), in which the red areas present ferrite phase and the green pixels indicate the austenite. To ensure the reliability of the measurements, the pixels with a confidence indexing (CI) less than 0.1 were discarded, and they appear in black on the map. The ACOM-TEM measurement confirms that austenite exists in some zones of the studied specimen and most of the austenite is located at the grain boundaries. The axis angle rotation boundaries with $90^{\circ}<112>$, representing the $\mathrm{K}-\mathrm{S}$ orientation relationship between austenite and martensite [8], are plotted in white. The enlarged views of the areas, indicated by the open square 1-3, are shown in Figure 5(b)-(d). It can be seen that the boundaries between austenite and adjacent ferrite fulfil the $\mathrm{K}-\mathrm{S}$ relationship.

\section{Discussion}

It is clear that the applied automatic orientation mappings are capable of characterizing the BEL in the studied rails. Correlations of BEL and WEL are indicated by the observations of similar lamellar features crossing the boundaries of the layers (BEL or WEL) and the pearlitic matrix [14]. As a consequence, nanometer size grains and zones with high dislocation densities are also possible within the BEL, referring to the microstructural features of WEL, $c f$. [2]. This can lead to difficulties of characterizing the BEL using orientation based microscopy technologies. Nevertheless, it is shown in this study that BEL is well characterized by the applied methods in the following aspects: (i) the IQ or IPF maps from all measurements reveal the elongated grain shapes within the characterized region of BEL, (cf. Figure 2(a) and (b)); (ii) with a relative larger scanned area in EBSD, grains aligned towards RD direction can be seen, e.g. Figure 2(b). Comparing with the undeformed lamellar pearlite microstructure inside the rail [11], the ferritic grains appear to be fragmented and refined; (iii) substructure-like features, having misorientation between $1-3^{\circ}$, are also clearly revealed by KAM (Figure 2(c), Figure 3(c) and Figure 4(c)). 


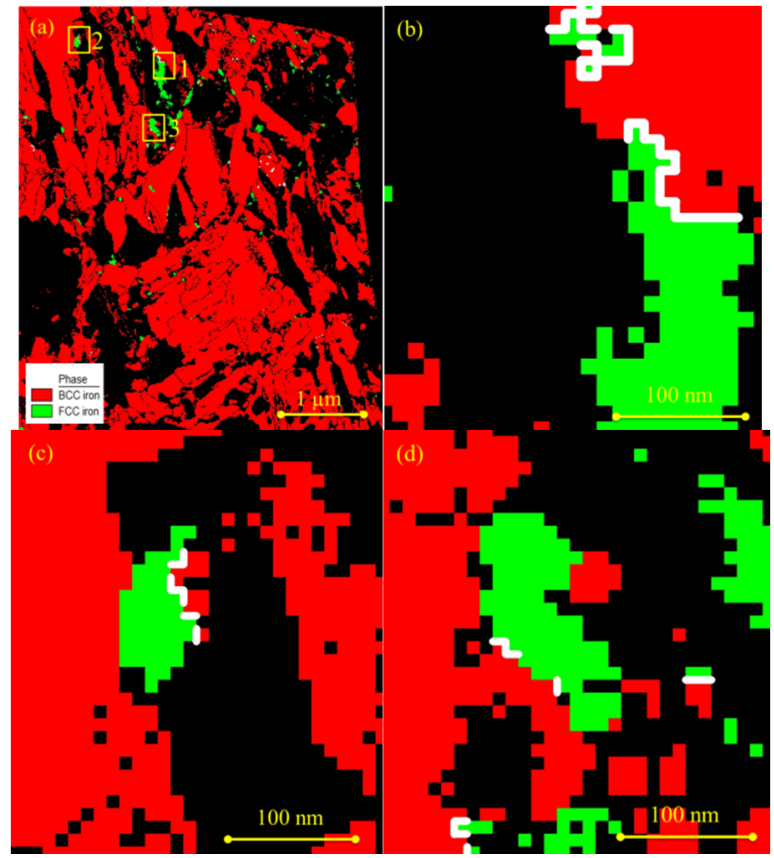

Figure 5: (a) Phase map of the ACOM-TEM scan on the FIB lamella. The red and green colours represent ferrite and austenite respectively. The $90^{\circ}<112>$ angle axis rotation angle represents the $\mathrm{K}-\mathrm{S}$ orientation relationship and is plotted by white lines; (b) enlarged view of the square area 1; (c) enlarged view of the square area 2; (d) enlarged view of the square area 3 . All enlarged views show the $\mathrm{K}-\mathrm{S}$ orientation relationship between ferrite/austenite.

ACOM-TEM provides more detailed information in characterizing the same FIB-lamella, in comparison with TKD. Extra microstructural features are identified by ACOM-TEM and one example is shown by comparing the features in the same grain with dashed-line inside the grain (see Figure 3(a) and Figure 4(a)). It can be seen that the grain in Figure 4(a) are further divided into several sub-units by narrow bright lines. The misorientation profiles along the respective dash-lines are shown in Figure 6(a) and Figure 6(b). The red line shows the point to point (PTP) misorientation and the blue line shows point to origin (PTO) misorientation between the individual pixels along the line. Clearly the misorientation between the start to end of the line is larger $\left(\sim 7^{\circ}\right)$ for ACOM-TEM than TKD $\left(<1.8^{\circ}\right)$. This is probably due to the smaller step size used for the ACOM-TEM, so that the sub-units are used and the cumulative rotations can be identified.

The current experimental results indicate the effect of plastic deformation on the microstructures within the BEL. The ferritic grains are seen to be fragmented (cf. IQ map in Figure 2(a), Figure 3(a) and Figure 4(a)). Furthermore, the 

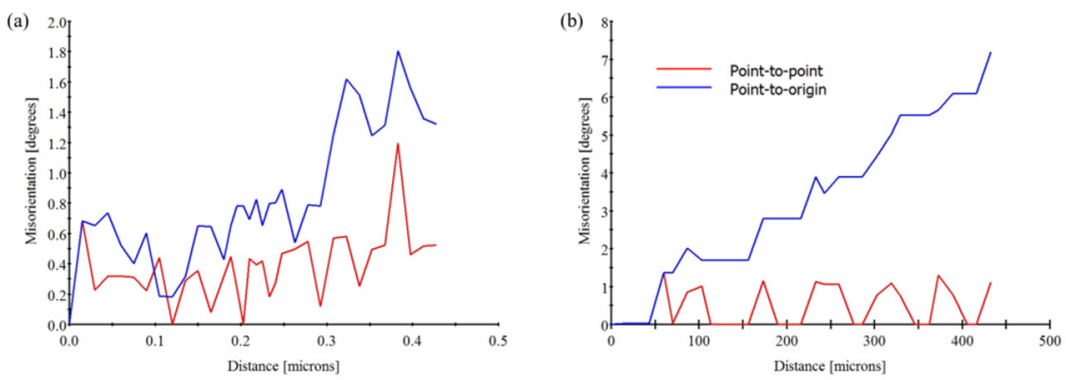

Figure 6: Misorientation profiles along the dash-line in the same grain from (a) TKD scan and (b) ACOM-TEM scan.

substructure-like features revealed by KAM, see e.g. Figure 2(c), are more likely the rearrangement of dislocations and might further develop into low angle grain boundaries. This evidence indicates the possible history of plastic deformation.

However, detection of retained austenite by ACOM-TEM, Figures 5(a)-(d), strongly indicates that the martensitic transformation has a contribution in formation of the BEL. The boundaries between the retained austenite and adjacent ferrite have the $\mathrm{K}-\mathrm{S}$ orientation relationship which is a strong indication of austenite to martensite transformation.

Current experimental evidence is not sufficient to clarify the nature of BEL. Further work should be done to support the current hypothesis: (i) The thermal history of the studied rail section during the past loadings should be studied. The temperature evolution can be used as evidence for supporting/excluding the phase transformation hypothesis. (ii) To prove or disprove the carbide precipitation in the BEL. From the morphology comparison, it appears that BEL might be correlated to WEL. Assuming that BEL has martensitic microstructure and considering the fact that BEL is significantly softer $(450 \mathrm{HV}-\max 752 \mathrm{HV})$ than the reported WEL with a hardness in the range between $700 \mathrm{HV}$ and $1200 \mathrm{HV}$ [14], it is very likely that BEL is the decomposition product of martensite. As a consequence, carbides are not observed at the used magnification in the orientation maps. Characterization by TEM at higher magnification and high resolution TEM will be excellent tool for the supporting this hypothesis.

\section{Summary}

In this work, the brown etching layer (BEL) is characterized by different types of automatic crystallographic orientation microscopy. It has been shown that EBSD, TKD and ACOM-TEM are suitable for characterizing the BEL phenomenon on different scales. Conventional EBSD is capable of characterizing the largest area whereas ACOM-TEM is the most powerful technique which allows the highest spatial resolution, compromising with the size of the scanned area. TKD fills the gap between these two techniques by potentially allowing a larger view field and 
excellent spatial resolution of $\sim 10 \mathrm{~nm}$. By combination of the three, the BEL is characterized at different resolution levels.

The grain refinement, grain adjustment towards loading direction and the substructures with low misorientations indicate the possible history of severe plastic deformation. The detection of retained austenite, having $\mathrm{K}-\mathrm{S}$ relationship with the adjacent BCC phase, strongly indicates the possibility of martensite in the BEL.

\section{Acknowledgements}

STW - The Netherlands and Prorail are gratefully acknowledged for the financial support in the frame of the project, PRIME (High Performance Rail through Intelligent Metallurgy \& Engineering). ProRail is acknowledged for supporting the research with providing the material and data for the study. Prof Dr Rolf Dollevoet is acknowledged for the interesting discussion and critical comments. Ir An Verdiere from Ghent University is acknowledged for her kind assistance in preparing the FIB lamella.

\section{References}

[1] Dikshit, V., Clayton, P. and Christensen, D. Investigation of rolling contact fatigue in a head-hardened rail. Wear, 1991, 144(1-2), pp. 89-102.

[2] Newcomb, S.B. and Stobbs, W.M. A transmission electron microscopy study of the white-etching layer on a rail head. Materials Science and Engineering, 1984, 66(2), pp. 195-204.

[3] Österle, W., Rooch, H., Pyzalla, A. and Wang, L. Investigation of white etching layers on rails by optical microscopy, electron microscopy, X-ray and synchrotron X-ray diffraction. Materials Science and Engineering: A, 2001, 303(1-2), pp. 150-157.

[4] Wild, E., Wang, L., Hasse, B., Wroblewski, T., Goerigk, G. and Pyzalla, A. Microstructure alterations at the surface of a heavily corrugated rail with strong ripple formation. Wear, 2003, 254(9), pp. 876-883.

[5] Wang, L., Pyzalla, A., Stadlbauer, W. and Werner, E.A. Microstructure features on rolling surfaces of railway rails subjected to heavy loading. Materials Science and Engineering: A, 2003, 359(1-2), pp. 31-43.

[6] Takahashi, J., Kawakami, K. and Ueda, M. Atom probe tomography analysis of the white etching layer in a rail track surface. Acta Materialia, 2010, 58(10), pp. 3602-3612.

[7] Lojkowski, W., Djahanbakhsh, M., Bürkle, G., Gierlotka, S., Zielinski, W. and Fecht, H.J. Nanostructure formation on the surface of railway tracks. Materials Science and Engineering: A, 2001, 303(1-2), pp. 197-208.

[8] Brückner, G., Pospiech, J., Seidl, I. and Gottstein, G. Orientation correlation during diffusional $\alpha \rightarrow \gamma$ phase transformation in a ferritic low carbon steel. Scripta Materialia, 2001, 44(11), pp. 2635-2640. 
[9] Morito, S., Tanaka, H., Konishi, R., Furuhara, T. and Maki, T. The morphology and crystallography of lath martensite in Fe-C alloys. Acta Materialia, 2003, 51(6), pp. 1789-1799.

[10] Zelin, M. Microstructure evolution in pearlitic steels during wire drawing. Acta Materialia, 2002, 50(17), pp. 4431-4447.

[11] Wu, J., et al. A microstructural study of rolling contact fatigue in rails. In: Proceedings of the Second International Conference on Railway Technology: Research, Development and Maintenance, Ajaccio, France, 811 April 2014. Civil-Comp Press.

[12] Trimby, P.W., et al. Characterizing deformed ultrafine-grained and nanocrystalline materials using transmission Kikuchi diffraction in a scanning electron microscope. Acta Materialia, 2014, 62(0), pp. 69-80.

[13] Wang, B., et al. Advanced TEM investigation of the plasticity mechanisms in nanocrystalline freestanding palladium films with nanoscale twins. International Journal of Plasticity, 2012, 37(0), pp. 140-156.

[14] Li, S., Wu, J., Petrov, R.H., Sietsma, J., Li, Z. and Dollevoet, R. 'Brown etching layer': a possible insight into the formation mechanism of white etching layer on rail steels? Manuscript in preparation, 2015. 